\title{
Calculation of $x$-ray spectra for nanocrystalline materials
}

\author{
P. M. Derlet, S. Van Petegem, and H. Van Swygenhoven \\ Paul Scherrer Institute, Villigen, CH-5232, Switzerland \\ (Received 15 September 2004; revised manuscript received 12 November 2004; published 26 January 2005)
}

\begin{abstract}
The calculation of two-theta x-ray diffraction profiles for multimillion atom computer generated nanocrystalline (nc) Ni systems is presented. Peak profile analysis is performed using a standard Williamson-Hall analysis. The derived mean grain size and root-mean-square inhomogeneous strain quantities are compared to similar quantities obtained by direct structural investigation and visualization of the nc atomic configurations. Debye-Waller factors are also calculated from the theoretical x-ray spectra and compare well with those derived from the phonon properties of the nc system.
\end{abstract}

DOI: 10.1103/PhysRevB.71.024114

PACS number(s): 61.46.+w, 61.10.-i, 07.05.Tp

\section{INTRODUCTION}

The structure and mechanical properties of fully dense nanocrystalline (nc) metals have become the focus of intense research activity in great part due to the tremendous increase in measured strength. ${ }^{1,2}$ Whether these materials plastically deform via the high density of interfaces through, for example, grain boundary (GB) sliding, via slip activity within the grains or a combination of both, is a matter of discussion. Atomistic simulation has indicated that when there is slip, it is initially nucleated at the GB by means of a partial dislocation and extends through the grain, either as a partial or full dislocation or possibly as a twin fault. Particularly, dislocations are absorbed in the surrounding GB network ${ }^{3-6}$ leaving no dislocation debris inside the grain. Simulation has also demonstrated, that apart from an increased triple junction density, the structure of the GBs are not so different from those observed in the polycrystalline regime. ${ }^{7-9}$

The use of $\mathrm{x}$-ray diffraction (XRD) techniques has played a central role in the microstructural characterization of nc materials, ${ }^{10,11}$ allowing a determination of mean grain size and distribution, and also root-mean-square (RMS) strain, through peak profile analysis. ${ }^{12,13}$ The presence of dislocations within grains and GBs contributes to the RMS strain, and thus XRD can in principle constitute a probe into the dislocation content of a material. This is particularly the case for the polycrystalline regime, where XRD profile analysis procedures have been developed to determine dislocation densities assuming lattice dislocations of a particular screw/ edge type. ${ }^{14,15}$ More recently, XRD has been used to study the plastic deformation properties of nc Ni via in situ deformation in the Swiss synchrotron Light Source (SLS), ${ }^{16}$ where a series of loading (beyond the yield point) and unloading experiments were performed during which the peak position and width were measured. For the coarse grained material, a peak broadening was observed during loading that is predominantly irreversible upon unloading due to a dislocation network that is built-up during plastic deformation, a well-known mechanism. However, for the nc Ni samples, the peak broadening is entirely recoverable upon unloading indicating that no permanent RMS strain is built-up during plastic deformation, and thus no permanent dislocation network develops. This observation is entirely compatible with the results of atomistic simulations, since full dislocations nucleated and absorbed in the GB network do not leave a lasting defect structure within the grains they transect.

The complexity of the nc system makes it difficult to develop an associated theoretical model of nc XRD peak profile behavior in terms of a specific nc microstructure. In this paper we therefore investigate the nontrivial effect of GB structure on RMS strain and size broadening by numerical simulation of XRD profiles derived from an atomic scale description of the nc environment. By considering nc samples under zero loading conditions we exploit the Debye assumption of structural isotropy to calculate the full twotheta powder diffraction profile for a typical synchrotron wavelength. We do this for two nc samples with respective average grain sizes of 5 and $12 \mathrm{~nm}$. Using the spectra, we then perform a Williamson-Hall analysis and correlate its predictions by exploiting the atomic scale resolution available to us. The XRD derived thermal component of the Debye-Waller factor is also determined and compared to values calculated via the simulated nc vibrational density of states. We conclude with a discussion of what constitutes an appropriate definition of the GB region in the context of XRD measurements.

\section{COMPUTATIONAL APPROACH}

We consider an $N$ atom configuration defined by its spatial coordinates $\mathbf{r}_{i}$ immersed entirely in a monochromatic plane wave $\mathrm{x}$-ray beam. The asymptotic scattered wave function for a given scattering vector $\mathbf{k}$ will be

$$
\psi_{\text {scatt }}(\mathbf{k})=\sum_{i}^{N} f_{i} \exp \left(i \mathbf{k} \cdot \mathbf{r}_{i}\right)
$$

where $f_{i}$ is the $\mathrm{x}$-ray atomic form factor for atom $i$. To obtain a standard two-theta spectrum, a spherical average of the scattering intensity is performed:

$$
I(k)=\frac{1}{4 \pi} \int d \Omega_{\hat{\mathbf{k}}}\left|\sum_{i} f_{i} \exp \left(i k \hat{\mathbf{k}} \cdot \mathbf{r}_{i}\right)\right|^{2},
$$

where $k=2 \pi \sin (2 \theta) / \lambda$. The spherical integration can be performed analytically, resulting in the well-known Debye scat- 
tering formula. ${ }^{12}$ Assuming a monoatomic system $\left(f_{i}=f\right)$, we obtain

$$
I(k)=|f|^{2}\left(N+\sum_{i, j, i \neq j}^{N} \frac{\sin k r_{i j}}{k r_{i j}}\right),
$$

where $r_{i j}=\left|\mathbf{r}_{i}-\mathbf{r}_{j}\right|$.

The computational advantage of performing the spherical average analytically is offset by Eq. (3) containing a summation over atomic pairs, scaling thus as $N^{2}$. An alternative approach would be to first numerically construct Eq. (1) for a given $k$ for a range of appropriate $\hat{\mathbf{k}}$ and then to perform the spherical average via a numerical integration using a spherical Gaussian quadrature scheme. Such an approach will scale linearly with $N$ and therefore is amenable to large systems. A disadvantage of this method lies in the choice of the Gaussian quadrature scheme since it must be accurate and efficient for a wide range of Bragg directions. Moreover, for the nc regime, where significant broadening is expected, good accuracy is needed over the entire scattering spectrum.

An intermediate computational solution can be found by recasting the double summation over atomic positions into a one-dimensional integration over bond lengths

$$
I(k)=|f|^{2}\left(N+\int_{0}^{\infty} d r l(r) \frac{\sin k r_{i j}}{k r_{i j}}\right),
$$

where $l(r) d r$ gives the number of bonds between length $r$ and $r+d r$. The problem thus reduces to determining $l(r)$ and for a given system this quantity is formally given by

$$
l(r)=\sum_{i, j, i \neq j} \delta\left(r-r_{i j}\right) .
$$

A double summation is again evident, however, computationally such a summand can be evaluated rapidly compared to the division, multiplication, and sinus evaluation needed in Eq. (3). Such an approach is found to work well for atomic systems with up to 10 million atoms.

For large $r$, it becomes difficult for $l(r)$ to resolve atomic structure, and $l(r)$ asymptotes to the continuum limit

$$
l(r) \sim \rho 4 \pi r^{2},
$$

where $\rho$ is the bulk density of the sample. Thus the integral in Eq. (4) can be split into

$$
\int_{0}^{r_{c}} d r l(r) \frac{\sin k r_{i j}}{k r_{i j}}+\rho 4 \pi \int_{r_{c}}^{\infty} d r r^{2} \frac{\sin k r_{i j}}{k r_{i j}},
$$

which reduces to

$$
\int_{0}^{r_{c}} d r l(r) \frac{\sin k r_{i j}}{k r_{i j}}+\rho 4 \pi \frac{\cos k r_{c}}{k^{2}} .
$$

Thus the computational problem is reduced to determining $l(r)$ only up to some continuum cut-off range $r_{c}$. Apart from the wavelength of the incident radiation, the value of $r_{c}$ will also depend on the material structure. For example, in weakly textured and equiaxed nc systems, $r_{c}$ will typically have a value between one to two grain diameters, providing for an effective linear scaling with system size when the corresponding characteristic grain size is much smaller.

\section{COMPUTER-GENERATED NANOCRYSTALLINE SAMPLE PREPARATION}

The three-dimensional nc-Ni structure is constructed by beginning with an empty simulation cell under full periodic boundary conditions and choosing randomly a number of positions that constitute the fcc grain centers. The number of grain centers is determined from the simulation cell size and the desired characteristic grain size. For each grain center a random orientation is chosen and an fcc lattice is constructed geometrically. At a point where atoms from one grain center are closer to the center of another grain, construction is halted. Eventually construction will cease throughout the entire sample, resulting in a three-dimensional granular structure according to the Voronoi construction. At this stage atom pairs, each atom originating from a different crystallite, are inspected and where there is a nearest-neighbor distance of less than $80 \%$ of the fcc nearest-neighbor distance, one atom is removed. Molecular statics is then performed to relax any local high potential energy configuration that may exist, followed by $100 \mathrm{ps}$ of $N-P-T$ molecular dynamics (MD) at room temperature to further relax the structure. All MD is performed within the Parrinello-Rahman ${ }^{17}$ approach with periodic boundary conditions and fixed orthorhombic angles. The simulations are carried out at room temperature. For the simulation of $\mathrm{Ni}$, we used the second moment tight-binding potential of Cleri and Rosato. ${ }^{18}$ A more detailed discussion of the preparation procedure and the issues associated with the resulting grain boundary structure and network topology, and its relation to experiment, can be found in Refs. 8 and 19

To identify the GB network structure a medium range environmental analysis is performed for each atom, where a local crystallinity class is assigned to each atom. ${ }^{20}$ Using this classification scheme, we presently define three classes of atoms: PFCC - atoms that are fcc coordinated up to fourth nearest neighbor, GFCC - atoms that are fcc coordinated up to first nearest neighbor, and GB-remaining atoms. It is the latter classification that allows easy identification of GB and the GB network structure.

For the calculation of $\mathrm{x}$-ray spectra, two samples are used, each containing approximately 1.2 million atoms. The $\mathrm{Ni}$

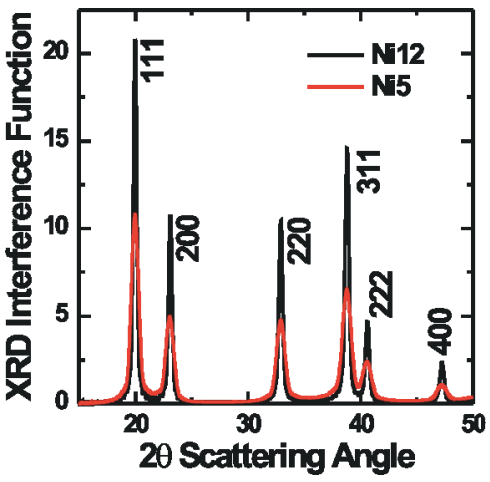

FIG. 1. (Color online) Calculated XRD spectra for Ni12 and Ni5 nc systems. 


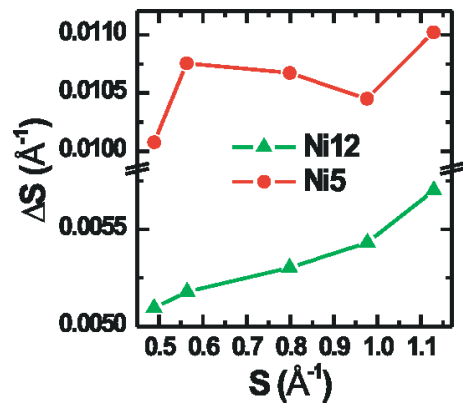

FIG. 2. (Color online) A Williamson-Hall plot of the Ni5 and Ni12 samples in terms of the scattering magnitude $s=\sin \theta / \lambda$.

samples have been used extensively in previous studies of grain boundary structure $7,9,21$ and the deformation properties of nc materials. ${ }^{22-24}$ The Ni12 sample contains 15 grains with an average grain diameter of $12 \mathrm{~nm}$ and the Ni5 sample contains 125 grains with an average grain diameter of $5 \mathrm{~nm}$. Both samples contain randomly selected grain orientations. Actual X-ray spectra for $\mathrm{Ni} 12$ and Ni5 where determined from both an instantaneous atomic configuration at $300 \mathrm{~K}$ and also an atomic configuration derived from an average of 1 ps of MD at $300 \mathrm{~K}$.

\section{Results}

\section{A. Peak profile analysis}

In what follows, we calculate the two-theta powder diffraction profile using Eq. (4) for a synchrotron photon energy of $17.5 \mathrm{keV}(\lambda=0.71 \AA)$ using the averaged computer generated nc configurations. We assume $|f|^{2}$ in Eq. (4) to be equal to unity for all scattering vector magnitudes and therefore the following spectra are in fact interference functions, however, we continue to refer to them as XRD spectra. Figure 1 displays the spectra for $\mathrm{Ni} 12$ and $\mathrm{Ni5}$, respectively, for the averaged atomic configurations. The angular range is chosen to include the (111) to (400) range of peaks. As expected the peaks are broader for Ni5 than Ni12.

Figure 2 displays the resulting $\Delta s$ integral peak widths as a function of scattering vector $s$ for the (111), (200), (220), (222), and (400) peaks for Ni5 and Ni12. A Williamson-Hall analysis assumes that if the RMS strain is isotropic (and therefore linearly dependent on the scattering order magnitude), then the points in Fig. 2 (a Williamson-Hall plot) will all fall on a straight line, the $y$ intercept of which will be inversely proportional to the volume averaged coherent scattering length - the volume averaged grain diameter. In Fig. 2 both the Ni12 and Ni5 samples exhibit some degree of anisotropic RMS strain.

The results of a Williamson-Hall analysis on the simulated spectra assuming a Cauchy-Cauchy (CC), GaussianGaussian (GG), and a Cauchy-Gaussian (CG) coherent volume/RMS strain convolution are shown in Table I. All three convolution assumptions produce approximately the same mean grain size, although the $\mathrm{CC}$ convolution gives a higher grain size as is generally the case. ${ }^{10}$ For the RMS strains there is a greater difference over the three convolutions. We note that the assumption of a Cauchy distribution for the coherent scattering lengths and a Gaussian distribution for the strain can be justified using physical arguments. ${ }^{13}$ The relative insensitivity of the mean grain size on the distribution assumption may be a result of the small number of grains in the samples, and that both a Cauchy or Gaussian distribution might fit such a discrete distribution of sizes equally well. We see that the grain size predictions are close to the known grain diameters of the two samples. It should be pointed out that the " 5 " and " 12 " nanometer sample sizes are to be considered more as labels, since in the original Voronoi construction, these numbers approximately represent the mean distance between the initial grain centers rather than true mean diameters, and that the Voronoi tessellation can produce a variety of polyhedra shapes that would be averaged out in the spherical integration used to produce the Debye spectra in Fig. 1.

Since the nanocrystalline samples contain defect-free grain interiors, the measured RMS strain must arise from elastic variations of the lattice parameter in the grain interiors due to the surrounding GB network. Figure 3 displays a cross section of both the 5 and $12 \mathrm{~nm}$ grain size samples in which the atoms are shaded according to the local crystallinity classification (GFCC and PFCC - gray and GB-black) and also colored (shaded) according to their local hydrostatic pressure in GPa [see caption and color (grayscale) bar in the figure]. In the present work, the local hydrostatic pressure is calculated via

$$
P_{\Omega}=\frac{1}{3 \Omega}\left\langle\sum_{i}\left(m v_{i}^{2} \Lambda_{i}+\frac{1}{2} \sum_{\langle j\rangle} \vec{F}\left(r_{i j}\right) \cdot \vec{r}_{i j} l_{i j}\right)\right\rangle,
$$

where $\Omega$ is now the volume of some region of interest. In Eq. (9), $\Lambda_{i}$ is unity if atom $i$ is within the volume element and zero otherwise, and $l_{i j}$ is the fraction of the length of the

TABLE I. Williamson-Hall (WH) grain diameter and RMS-strain results assuming a Cauchy-Cauchy (CC), Gaussian-Gaussian (GG), and Cauchy-Gaussian (CG) convolution for the coherent volume/RMS strain.

\begin{tabular}{lccccc}
\hline \hline & \multicolumn{3}{c}{$5 \mathrm{~nm}$} & & \multicolumn{2}{c}{$12 \mathrm{~nm}$} \\
\cline { 2 - 3 } \cline { 5 - 6 } & $D(\mathrm{~nm})$ & $\overline{\delta \varepsilon^{2}}(\%)$ & & $D(\mathrm{~nm})$ & $\overline{\delta \varepsilon^{2}}(\%)$ \\
\hline WH-CC & $5.6 \pm 0.2$ & $0.19 \pm 0.04$ & & $10.6 \pm 0.2$ & $0.07 \pm 0.01$ \\
WH-GG & $5.2 \pm 0.1$ & $0.49 \pm 0.06$ & & $10.0 \pm 0.1$ & $0.22 \pm 0.02$ \\
WH-CG & $5.3 \pm 0.2$ & $0.41 \pm 0.09$ & & $10.2 \pm 0.1$ & $0.18 \pm 0.02$ \\
\hline \hline
\end{tabular}



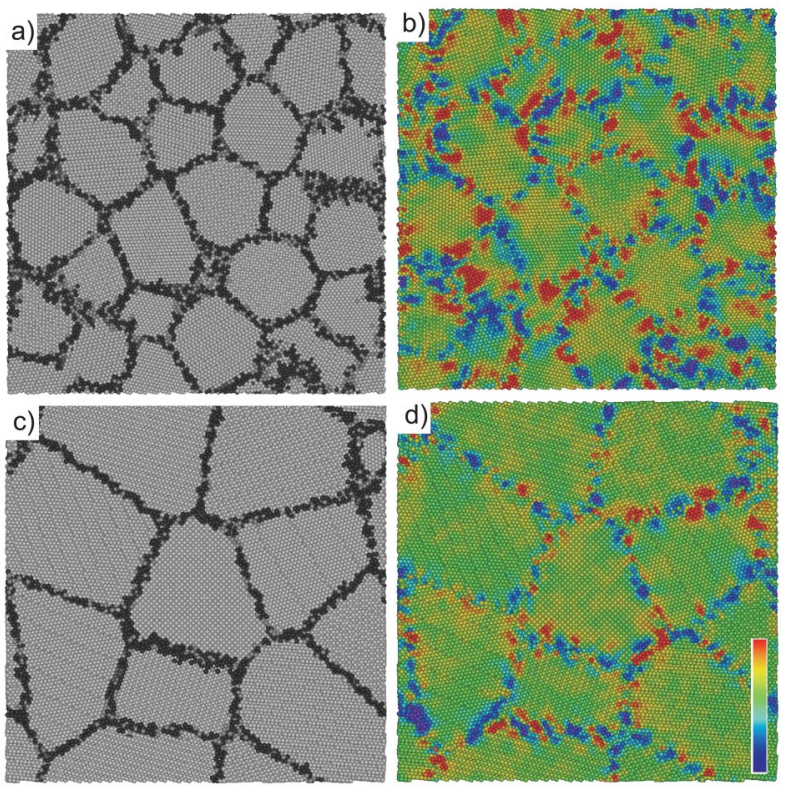

FIG. 3. (Color online) (a) and (c) are cross sections of the Ni5 and Ni12 nc samples with atoms colored (shaded) according to their local crystallinity. (b) and (d) are the same cross sections with the atoms colored (shaded) according to their local hydrostatic pressure. Here red (light gray) represents atoms with greater than $2 \mathrm{GPa}$ and blue (dark gray) less than $-2 \mathrm{GPa}$ hydrostatic pressure; see color (grayscale) bar.

bond between atoms $i$ and $j$ that lies within the volume element. Equation (9) is an extension of the virial theorem and rigorously satisfies conservation of linear momentum for the chosen volume. ${ }^{25}$ In the present work the volume element is chosen to be a sphere of radius $4 \AA$ centered on each atom. Here \langle\rangle represents a 500 femtosecond time average. For more details of Eq. (9) being applied to the nc regime see Ref. 26. In the present work, a positive pressure value represents local compression and a negative value represents local tension.

Inspection of Fig. 3 reveals that there is significant pressure variation at the GB regions, often with alternating patterns of local compressive and tensile regions. Extensive investigations into the structure of the GB of the computergenerated nc samples has revealed that many of the GBs can be visualized via an intrinsic GB dislocation network or equivalently regions of misfit and coherency. ${ }^{7-9,22}$ The strong stress variations seen in Fig. 3 generally correlate with such GB dislocation networks and are expected to be the main source of RMS strain anisotropy in the computer-generated nc samples. This is reflected in Williamson-Hall plots for Ni12 and Ni5 (Fig. 2) where RMS strain anisotropy is clearly evident, more so in the Ni5 sample due to the larger interface density.

Figure 4 now displays the local hydrostatic pressure distribution for only the fcc atoms for both the Ni5 and Ni12 samples, and we see that for the Ni5 sample, the distribution is broader. Indeed the standard deviation of the Ni5 sample is $1.061 \mathrm{GPa}$ where as for the Ni12 sample it is $0.695 \mathrm{GPa}$. When one assumes a local elastic model for the stress/strain relationship, the local energy as a function of isotropic strain will be quadratic whereas the volume expansion will of

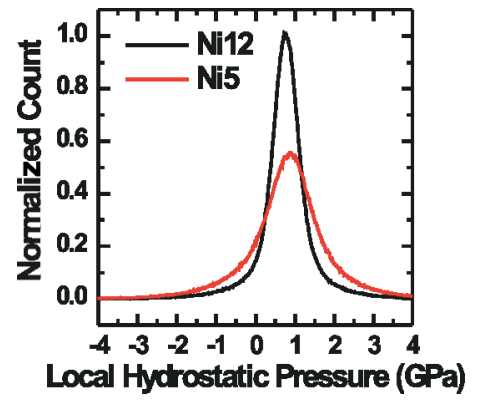

FIG. 4. (Color online) Local pressure distribution of the grain interiors of the Ni5 and Ni12 samples.

course be linear. Since $P=-\delta U / \delta V$ ( $U$ is the internal energy and $V$ its volume), the local hydrostatic pressure should then scale linearly with the local isotropic strain and the ratio of local pressure variances for $\mathrm{Ni5}$ and $\mathrm{Ni12}$ will equal the square root of the ratio of the corresponding RMS strains. From Table I the latter ratios are 1.19, 1.49, and 1.51 for the $\mathrm{CC}$, GG, and CG convolutions, whereas from Fig. 4 the ratio of the local pressure standard deviations is 1.53. Thus the Williamson-Hall analysis produces a strain that is in approximate agreement with the internal grain pressure distribution of the sample.

Figure 4 suggests that the grain interiors are under positive local hydrostatic pressures of $0.83 \mathrm{GPa}$ for $\mathrm{Ni} 5$ and $0.76 \mathrm{GPa}$ for Ni12. The mean pressures are, however, not the correct values since these values represent the local pressure of a sphere of radius $4 \AA$ and not the bulk pressure of a grain-to calculate grain pressures much larger volumes are needed that approach the typical grain size of the system. Past work has revealed that the usual virial form of the local pressure represents the more correct macroscopic pressure of strained regular lattices, ${ }^{23}$ giving a value for the mean hydrostatic pressure of approximately $80 \mathrm{MPa}$ for Ni5 when only fcc coordinated atoms are considered. Thus the grains are under a net compressive pressure. Using the same method as in Ref. 23, the present configurations give an average grain (fcc) pressure of $130 \mathrm{MPa}$ for Ni5 and $80 \mathrm{MPa}$ for Ni12. In the case of Ni5 this differs from the earlier work of Ref. 23. Differences of the order of $10 \mathrm{~s}$ of $\mathrm{MPa}$ (at the 500 femtosecond time scale) are, however, to be expected given that the standard deviation of the stress distributions is of the order of GPa's.

Such compressive pressures for the grains entail a peak shift to smaller values relative to the fcc crystalline case. Using a simple linear isotropic elastic model $(P=B \varepsilon$ with $B$, the Bulk modulus for $\mathrm{Ni}$, taken as $180 \mathrm{GPa}$ ) the measured virial hydrostatic pressures correspond to isotropic strains of $0.07 \%$ for $\mathrm{Ni5}$ and $0.04 \%$ for Ni12. Inspection of the peak positions in Fig. 1 reveals that the peaks of Ni12 and Ni5 do move to the left and with corresponding $\varepsilon_{h k l}$ magnitudes similar to that predicted by this simple model. In the calculation of the peak shift, the bulk fcc peak positions were obtained from the Bragg formula using the $300 \mathrm{~K}$ equilibrium lattice constant $(3.544 \AA)$ associated with the CleriRosato Ni potential.

\section{B. Thermal effects}

Figures 5(a) and 5(b) display the spectra for Ni12 and Ni5 for both the averaged atomic configurations and also the in- 


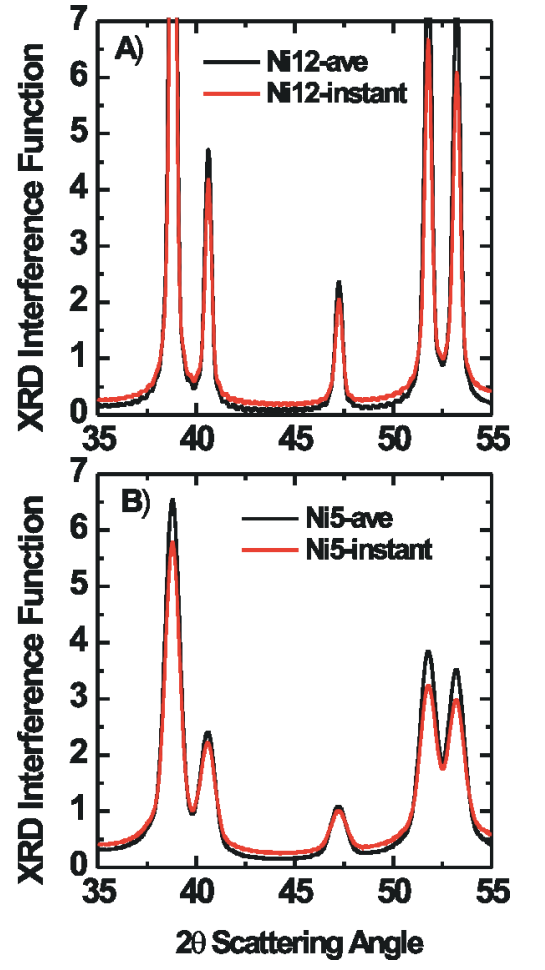

FIG. 5. (Color online) XRD spectra for thermally averaged and instantaneous configurations for (a) Ni5 and (b) Ni12.

stantaneous configurations, and we see that the peak heights of the diffraction peaks for the instantaneous configurations are somewhat reduced. This can be understood from the perspective of the ensemble of thermal displacements away from their average atomic positions that are stored within the instantaneous atomic nc configurations leading to a peak height reduction by an amount described by the DebyeWaller factor, $\exp (-2 W)$. From ${ }^{12}$

$$
\exp (-2 W)=\exp \left(-\frac{16 \pi^{2} \sin ^{2} \theta\left\langle u_{x}^{2}\right\rangle}{3 \lambda^{2}}\right)
$$

where $\theta$ is the peak position, $\lambda$ the wavelength of the incident radiation, and $\left\langle u_{x}^{2}\right\rangle$ is the atomic mean-squaredisplacement (MSD) along one coordinate direction. Thus by plotting $-\log \left(h_{i} / h_{a}\right)$ versus $\sin ^{2} \theta$, where $h_{i}\left(h_{a}\right)$ is the corresponding instantaneous (average) peak height, a linear relation is expected, the gradient of which will give $\left\langle u_{x}^{2}\right\rangle$. For Ni12 and Ni5 such a plot does exhibit good linear behavior, resulting in respective values of $42 \times 10^{-4}$ and $46 \times 10^{-4} \AA^{2}$ for $\left\langle u_{x}^{2}\right\rangle$ (see also Table II). These values compare well to the experimentally accepted room temperature value for bulk fcc $\mathrm{Ni},{ }^{27}$ which is $42 \times 10^{-4} \AA^{2}$.

An alternative approach to calculating the MSD can be found via the harmonic approximation of thermal vibrations (first given by Blackman ${ }^{28}$ ), where the atomic MSD along one coordinate direction can be determined directly from the (phonon) vibrational density of states (VDOS), $N(E)$ :
TABLE II. The mean-square displacements of thermal atomic vibrations calculated via XRD, simulated VDOS, and the experimental VDOS.

\begin{tabular}{lccc}
\hline \hline & XRD $\left(\times 10^{-4} \AA^{2}\right)$ & MD $\left(\times 10^{-4} \AA^{2}\right)$ & Calculated $\left(\times 10^{-4} \AA^{2}\right)$ \\
\hline fcc & & 43 & 42 \\
Ni12 & 42 & 52 & \\
Ni5 & 46 & 59 & \\
GB & & 85 & \\
GFCC & 51 \\
PFCC & 44 \\
\hline \hline
\end{tabular}

$$
\left\langle u_{x}^{2}\right\rangle=\frac{\hbar^{2}}{2 m} \int d E \operatorname{coth}\left(\frac{E}{2 k_{b} T}\right) \frac{N(E)}{E} .
$$

The VDOS for a particular atom can be obtained via the Fourier transform of the atomic velocity autocorrelation function, which in turn can be obtained by performing MD at a chosen temperature (in this case $300 \mathrm{~K}$ ). The bulk VDOS is then found from the average VDOS of all atoms in the system. For more details see Ref. 8. Figure 6(a) displays the resulting VDOS for $\mathrm{Ni5}, \mathrm{Ni12}$, and also for a crystalline $\mathrm{Ni}$ sample of similar size, and demonstrates that with decreasing grain size there is an increasing enhancement of the VDOS at low and high phonon energies and a broadening of the van Hove peaks. ${ }^{8,29}$ Table II lists the resulting MSDs for the three VDOS in Fig. 6 using Eq. (11), along with those derived from the XRD spectra in Fig. 5, and also the accepted experimental value for fcc $\mathrm{Ni}^{27}$ Both the XRD and MD derived values for $\mathrm{Ni} 5$ and $\mathrm{Ni} 12$ demonstrate an increase relative to the fcc value with decreasing grain size, but for the MD value this increase is greater.

Recently it has been established that the nc VDOS can be directly decomposed into a contribution due to the GB region and a contribution due to the grain interior, the fractions of which depend on the relative volumes of GB network and grains. ${ }^{30}$ Figure 6(b) displays the VDOS for PFCC, GFCC, and GB atoms, the forms of which depend little on the grain size of the system. One can see that the grain interior VDOS is similar to the fcc VDOS and that the GB VDOS exhibits a less structured form without strong van Hove peaks. Thus the bulk low and high frequency enhancements arise directly from the GB VDOS component. Using Eq. (11) the MSDs of these partial VDOSs are calculated and listed in Table II. We see that the GB MSD is considerably larger than that for the PFCC and GFCC local atomic environments. We note that the harmonic approximation inherent in Eq. (11) works well for a regular lattice, however, it is expected to give less accurate results for disordered regions such as GB where anharmonic (phonon-phonon) effects become increasingly important.

An important observation is that the XRD derived values for Ni12 and Ni5 are somewhat less than the values derived via MD, and not so different from the known calculated fcc value. The origin of this result lies in the XRD technique probing those atoms that contribute to the height of the Bragg peaks and therefore the coherent scattering component 


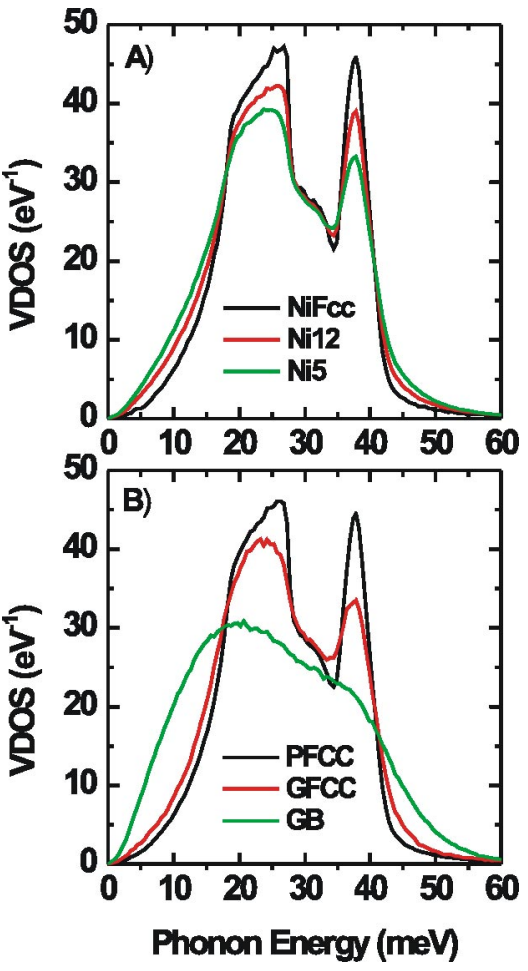

FIG. 6. (Color online) (a) The VDOS for fcc Ni and nc-Ni as a function of grain size. (b) The partial nc VDOSs for PFCC, GFCC, and GB atoms.

of the spectrum. Thus the XRD derived MSD primarily reflects the MSD of the nc grain interiors, and not that of the GB which will contribute more to the incoherent scattering component of the spectrum. This is evident in Table II where the PFCC MSD is similar in value to that seen in the XRD result.

\section{DISCUSSION AND CONCLUDING REMARKS}

By calculating XRD spectra of computer-generated nc samples we are able to correlate the predictions of wellknown profile analysis techniques with the atomic scale knowledge available to us by atomistic simulation methods. It, however, becomes clear that when considering the grain and GB properties, the definition of what constitutes a GB becomes an important concern.

Figure 7 displays a typical general high angle GB in Ni12 with the viewing direction along the GB plane (this GB has been investigated in detail in Fig. 9 in Ref. 21). The yellow (gray) scale bar is approximately $2 \mathrm{~nm}$ in length. The shading in Fig. 7(a) is in terms of the usual crystallinity definition, where the gray atoms represent fcc coordinated atoms and the darker shaded atoms represent non-fcc coordinated atoms. According to this definition we estimate the thickness of the GB as about $1 \mathrm{~nm}$. In Fig. 7(b) the atoms are now colored (shaded) according to Eq. (9), as in Fig. 3, and we see that the GB consists of alternating regions of compressive and tensile pressure that extend into the grains beyond the $1 \mathrm{~nm}$ estimate derived from the crystallinity classification. Thus according to the local hydrostatic pressure, the GB
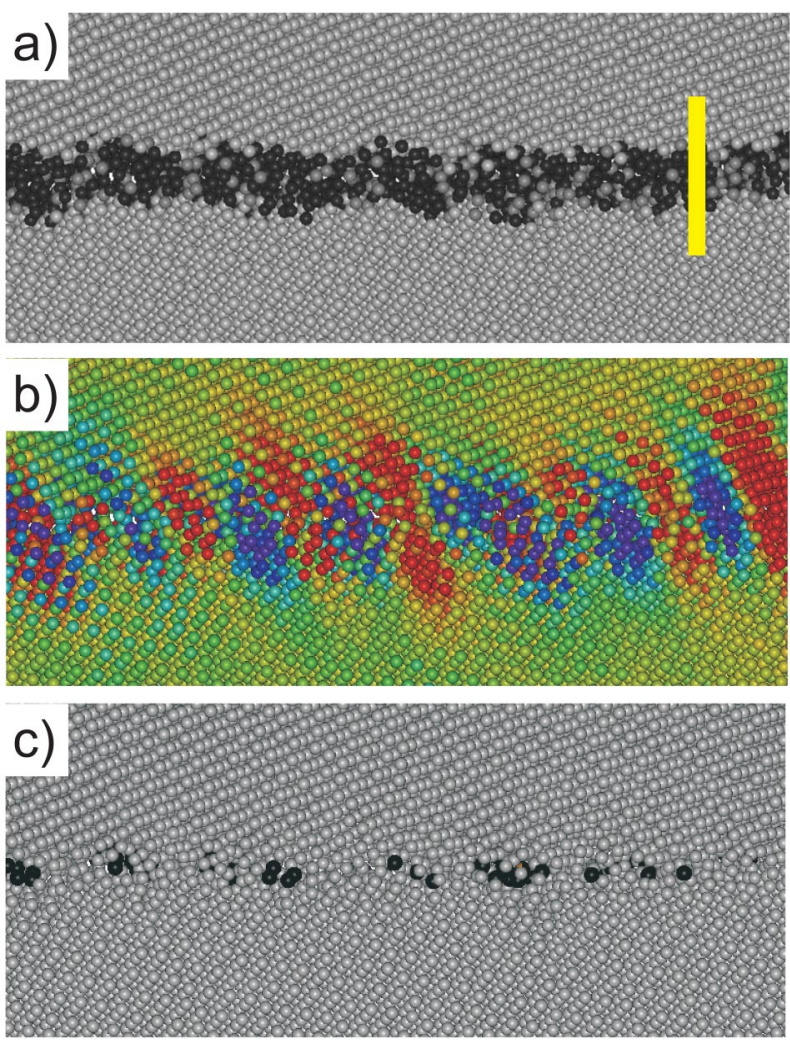

FIG. 7. (Color online) A general high angle grain boundary with atoms represented by (a) local crystallinity, (b) local hydrostatic pressure, and (c) position disorder.

thickness might be between 2 and $3 \mathrm{~nm}$, the precise thickness depending on the pressure cutoff criterion used. Past work has revealed that the fcc classification according to Honeycutt and Anderson ${ }^{20}$ omits atoms at the interface regions that are located at an fcc lattice site associated with one of the neighboring fcc grains: ${ }^{9}$ the so-called positionally ordered atoms. Figure 3(c) now displays atoms according to their positional order/disorder, where black atoms represent positionally disordered atoms whose location cannot be identified with the fcc lattice of either grain. Using this definition, the GB now has a thickness of the order of a lattice constant.

The question now arises as to which is the most appropriate definition or thickness criterion for a GB from the perspective of an XRD measurement. In the present work, when using the classification of a GB as those atoms belonging to the non-fcc class (as defined by Honeycutt and Anderson ${ }^{20}$ ), the XRD microstructural and thermal predictions appear to correlate well with direct measurement by atomistic methods. An alternative approach might be to consider only positional disordered atoms as those defining the GB region [Fig. 7(c)]. If we recalculate the mean virial hydrostatic pressure assuming all positionally ordered atoms now belong to the grain interior we obtain $10 \mathrm{MPa}$ for Ni12 and $15 \mathrm{MPa}$ for Ni5, which is a reduction by nearly an order of magnitude when compared to the grain pressures using the fcc classification. Such changes will lead to predicted peak shifts that are smaller, also by an order of magnitude, than that seen for the calculated XRD patterns in Sec. IV. Moreover, the resulting VDOS derived grain MSD will be larger than that seen 


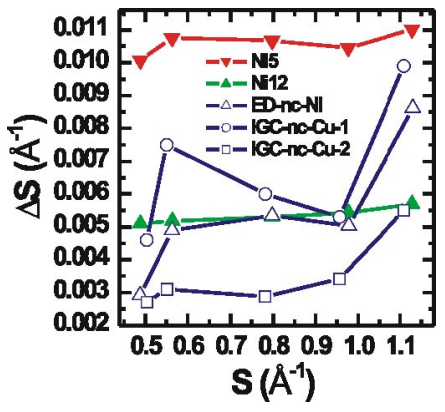

FIG. 8. (Color online) A Williamson-Hall plot of electrodeposited nc Ni (Ref. 16) and inert gas condensed nc Cu (Ref. 35), along with the simulated XRD data of the Ni5 and Ni12 samples.

by XRD, since the VDOS for positionally ordered and disordered (non-fcc) GB atoms is approximately the same due to strong nearest and next nearest-neighbor interactions. ${ }^{31}$ This indicates that those non-fcc GB atoms that are positionally ordered are strongly strained and therefore contribute primarily to the incoherent scattering component (the static Debye-Waller factor). This is evidenced in Figs. 7(a) and 7(b), where even fcc atoms that neighbor the (non-fcc) GB atoms experience strong pressure and therefore strain variations, but probably to a lesser extent that still allows them to contribute to the coherent component of scattered wave. Thus the non-fcc GB classification as defined by Honeycutt and Anderson ${ }^{20}$ appears to be more appropriate for agreement between the XRD derived and atomistic derived quantities.

Inspection of Fig. 2 and Table I demonstrates that if an XRD spectrum were calculated for a similarly generated computer nc sample with a mean grain size of 20-30 nm, the corresponding RMS strain is expected to become lower than what is experimentally observed. Indeed, real nc materials in the $20-30 \mathrm{~nm}$ grain size regime typically exhibit RMS strains that range between $0.1 \%$ and $0.4 \%$ for, respectively, inert-gas-condensed and electrodeposited synthesized nc materials. ${ }^{10,16,32-34}$ Figure 8 displays experimental Williamson-Hall plots for electrodeposited nc Ni (Ref. 16) and inert gas condensed $\mathrm{nc} \mathrm{Cu},{ }^{35}$ together with the calculated XRD spectra of samples Ni12 and Ni5. For nc-Ni (ED-nc$\mathrm{Ni}$ ) the data was obtained from a synchrotron x-ray light source with a wavelength equal to that used in the present simulation, while for $\mathrm{nc}-\mathrm{Cu}$ (IGC-nc-Cu-1) the data was measured using a conventional $\mathrm{x}$-ray machine. The experimental data (ED-nc-Ni and IGC-nc-Cu-1) display strong nonmonotomic behavior. Nevertheless, performing a Williamson-Hall analysis on the experimental data for ED-nc-Ni using only the 111 family of peaks, yields a 111 coherent scattering length of approximately $28 \mathrm{~nm}$ and a 111 RMS strain of approximately $0.4 \%$. A similar analysis for IGC-nc-Cu-1 results in a 111 scattering volume of $24 \mathrm{~nm}$ and 111 RMS strain of $0.1 \%$. In both cases, the scattering length agrees well with the average grain size as determined from TEM analysis. ${ }^{16,35}$ Compared to the simulated data for $\mathrm{Ni5}$ and Ni12, the experimental data is considerably more anisotropic indicating the possible presence of twins and/or anisotropic RMS strain dependencies due to specific dislocation/defect strain signatures that are not present in the current computer-generated grain boundaries. For example, the authors in Ref. 35 performed a simultaneous modified Williamson-Hall ${ }^{36}$ and Warren ${ }^{12}$ analysis that, respectively, take into account the XRD contrast factors of lattice dislocations and also the presence of twin faults within the grains, resulting in a twin faulting probability of $\beta=0.0442 \pm 0.002$. In Fig. 8, the data IGC-nc-Cu-2 represents the usual Williamson-Hall plot but now corrected from IGC-nc-Cu-1 for the planar twin faults. We see that the nonmonotomic behavior is now considerably reduced, but still larger than that which would be obtained for a $20-30 \mathrm{~nm}$ grain size version of $\mathrm{Ni5}$ and $\mathrm{Ni12}$, indicating that in addition to twins the experimental samples contain structural properties that are not present in the computer samples generated for the current work.

This result demonstrates that the comparison of the current simulated XRD data with experimentally derived XRD data must be taken with care since the microstructure of the experimental samples is not fully represented by the computer-generated structures, which do not contain extrinsic GB dislocations, lattice dislocations within grains, or twins. In terms of simulation, the nc sample preparation procedure involves only $100 \mathrm{ps}$ of $\mathrm{MD}$ at $300 \mathrm{~K}$, excluding any long range diffusion through which the GB might relax to a more equilibrium Burgers vector distribution with a correspondingly different stress signature. Moreover, it can be expected that impurities incorporated in the lattice structure of the grain and/or GB regions will also contribute to inhomogeneous strains. Experimental nc samples are not impurityfree with non-negligible concentrations of $\mathrm{C}, \mathrm{H}, \mathrm{Co}$, and $\mathrm{S}$, which may either remain within the grain interior or segregate to the GB region (see, for example, Ref. 34). To which degree the above-mentioned microstructural details will contribute to the RMS strain and strain anisotropy represented in a Williamson-Hall analysis needs to be addressed with further research.

In conclusion, the interpretation of x-ray measurements of nanoscale structures where the grain and grain boundary regions approach comparable orders of scale will be greatly facilitated by atomistic simulation, since it is precisely at this length scale that complete atomic scale resolution can be achieved to describe via molecular dynamics simulations nanocrystalline structures. The larger root-mean-square strains seen in the experimental nc Ni X-ray spectra indicate, however, that the computer-generated nc configurations do differ from experimental microstructures either in terms of the precise grain boundary/lattice dislocation content and/or impurity content. The presented technique will motivate the development of candidate nanocrystalline structures that contain preselected lattice dislocation structures, planar default structures such as twins and stacking faults, chemical impurities, and intrinsic and extrinsic grain boundary dislocation structures. It is indeed the strength of the current approach that computer-generated nanocrystalline structures can now be characterized using x-ray diffraction and therefore directly compared to the experimental regime, and through this a better characterization of experimental nanocrystalline structures can be obtained. 
${ }^{1}$ J. R. Weertman, Mechanical Behavior of Nanocrystalline Metals, Vol. Nanostructured Materials: Processing, Properties and Potential Applications (William Andrew, Norwich, NY, 2002).

${ }^{2}$ K. S. Kumar, H. Van Swygenhoven, and S. Suresh, Acta Mater. 51, 5743 (2003).

${ }^{3}$ J. Schiøtz and K. W. Jacobsen, Science 301, 1357 (2003).

${ }^{4}$ H. Van Swygenhoven, Science 296, 66 (2002).

${ }^{5}$ H. Van Swygenhoven, P. M. Derlet, and A. G. Frøseth, Nat. Mater. 3, 399 (2004).

${ }^{6}$ A. G. Frøseth, H. Van Swygenhoven, and P. M. Derlet, Acta Mater. 52, 2259 (2004).

${ }^{7}$ H. Van Swygenhoven, D. Farkas, and A. Caro, Phys. Rev. B 62, 831 (2000).

${ }^{8}$ P. M. Derlet, A. Hasnaoui, and H. Van Swygenhoven, Scr. Mater. 49, 629 (2003).

${ }^{9}$ P. M. Derlet and H. Van Swygenhoven, Phys. Rev. B 67, 014202 (2003).

${ }^{10}$ C. E. Krill and R. Birringer, Philos. Mag. A 77, 621 (1998).

${ }^{11}$ J. Weissmüller, J. Löffler, and M. Kleber, Nanostruct. Mater. 6, 105 (1995).

${ }^{12}$ B. E. Warren, X-Ray Diffraction (Addison-Wesley, Reading, 1969).

${ }^{13}$ H. P. Klug and L. E. Alexander, X-ray Diffraction Procedures: For Polycrystalline and Amorphous Materials, 2nd ed. (Wiley, New York, 1974).

${ }^{14}$ M. A. Krivoglaz, X-ray and Neutron Diffraction in Nonideal Crystals (Springer, Berlin, 2001).

${ }^{15}$ T. Ungar, J. Gubicza, G. Ribarik, and A. Borbely, J. Appl. Crystallogr. 34, 298 (2001).

${ }^{16}$ Z. Budrovic, H. Van Swygenhoven, P. M. Derlet, S. Van Petegem, and B. Schmitt, Science 304, 273 (2003).

${ }^{17}$ M. Parrinello and A. Rahman, J. Appl. Phys. 52, 7182 (1981).

${ }^{18}$ F. Cleri and V. Rosato, Phys. Rev. B 48, 22 (1993).
${ }^{19}$ H. Van Swygenhoven, P. M. Derlet, Z. Budrovic, and A. Hasnaoui, Z. Metallkd. 94, 1106 (2003).

${ }^{20}$ J. D. Honeycutt and H. C. Andersen, J. Phys. Chem. 91, 4950 (1987).

${ }^{21}$ H. Van Swygenhoven and P. M. Derlet, Phys. Rev. B 64, 224105 (2001).

${ }^{22}$ H. Van Swygenhoven, P. M. Derlet, and A. Hasnaoui, Phys. Rev. B 66, 024101 (2002).

${ }^{23}$ A. Hasnaoui, H. Van Swygenhoven, and P. M. Derlet, Acta Mater. 50, 3927 (2002).

${ }^{24}$ A. Hasnaoui, H. Van Swygenhoven, and P. M. Derlet, Phys. Rev. B 66, 184112 (2002).

${ }^{25}$ J. Cormier, J. M. Rickman, and T. J. Delph, J. Appl. Phys. 89, 99 (2001).

${ }^{26}$ M. Samaras, P. M. Derlet, H. Van Swygenhoven, and M. Victoria, Phys. Rev. B 68, 224111 (2003).

${ }^{27}$ L. M. Peng, G. Ren, S. L. Dudarev, and M. J. Whelan, Acta Crystallogr., Sect. A: Found. Crystallogr. 52, 456 (1996).

${ }^{28}$ M. Blackman, Proc. Cambridge Philos. Soc. 33, 380 (1937).

${ }^{29}$ P. M. Derlet and H. Van Swygenhoven, Phys. Rev. Lett. 92, 035505 (2004).

${ }^{30}$ P. M. Derlet, R. Meyer, J. L. Lewis, U. Stuhr, and H. Van Swygenhoven, Phys. Rev. Lett. 87, 205501 (2001).

${ }^{31}$ P. M. Derlet, S. Van Petegem, and H. Van Swygenhoven (unpublished).

${ }^{32}$ J. A. Eastmann, M. A. Beno, G. S. Knapp, and L. J. Thompson, Nanostruct. Mater. 6, 543 (1995).

${ }^{33}$ K. Reimann and R. Würschum, J. Appl. Phys. 81, 7186 (1997).

${ }^{34}$ F. Dalla Torre, H. Van Swygenhoven, and M. Victoria, Acta Mater. 50, 3957 (2002).

${ }^{35}$ T. Ungar, S. Ott, P. G. Sanders, A. Borbely, and J. R. Weertman, Acta Mater. 46, 3693 (1998).

${ }^{36}$ T. Ungar and A. Borbely, Appl. Phys. Lett. 69, 3173 (1996). 